\title{
RR charges of D2-branes in group manifold and Hanany-Witten effect
}

\author{
Takahiro Kubota ${ }^{a)}$ *and Jian-Ge Zhou ${ }^{b)} \dagger$
}

September 15, 2021

\begin{abstract}
a) Department of Physics, Graduate School of Science, Osaka University, Toyonaka, Osaka 560-0043, Japan

b) Institut für Theoretische Physik, Technische Universität Wien, Wiedner Hauptstrasse 8-10, A-1040 Wien, Austria
\end{abstract}

\begin{abstract}
By exploiting the correspondence between the Cardy boundary state in $S U(2)$ group manifold and the BPS D3-brane configuration in the full asymptotically flat geometry of NS5-branes, we show that the Hanany-Witten effect in 10D background is encoded in the Cardy boundary states. The two RR Page D0 charges of the $n$-th spherical D2-brane due to the contraction to $e$ or $(-e)$ is interpreted, and attributed to the Hanany-Witten effect.
\end{abstract}

*e-mail: kubota@het.phys.sci.osaka-u.ac.jp

†e-mail: jgzhou@hep.itp.tuwien.ac.at 
After the work of Bachas, Douglas and Schweigert [1] the issues of RR D0 charge and $U(1)$ flux quantization for the spherical D2-branes in $S U(2)$ group manifold have attracted much interest [2]-[11]. In [1], they calculated the RR charges of the spherical D2-branes via two approaches: one based on the Dirac-Born-Infeld action with WZ term; the other exploiting the exact 1-point functions in the boundary WZW model, and found that the RR charges of D2-branes are irrational in two calculations, but the $U(1)$ flux is quantized. In [6] and [10], it was pointed out that the Abelian Chern-Simons terms in the dynamics of the gauge fields, for instance, in massless type IIA supergravity, induce the modified Bianchi identity

$$
d \tilde{F}_{4}+F_{2} \wedge H_{3}=0
$$

where $\tilde{F}_{4}, F_{2}, H_{3}$ are gauge invariant field strengths of rank 4,2 , 3, respectively, and make the definition of charge in a gauge theory be more subtle. At least there are three natural notions of charge in a theory with Chern-Simons term: 'brane source charge', 'Maxwell charge' and 'Page charge', and it is Page charge, which corresponds to $U(1)$ flux, that should be quantized.

In [8] and [11, it was shown that in $S U(2)$ WZW model there are two different RR Page D0 charges 円 for the spherical D2-brane due to two contraction: either to $e$ or $(-e)$, and argued that the RR Page D0 charge should be only defined modulo $k$. In [8], it was expected this new feature should contain some important dynamical information, probably related to the fact that the $H$-field belongs to a nontrivial cohomology class.

In the present paper, we further explore the origin how two different $R R$ Page D0 charges for D2-brane are induced from the 10D dynamical point of view. In doing so, we consider the BPS D3-brane in the full asymptotically flat geometry of NS5-branes. Based on the numerical calculations in [9], we observe there is one to one correspondence between the BPS D3-brane configuration with $z_{\max } \longrightarrow \infty$ and opening angle $\psi_{n}$, and the $n$-th Cardy

\footnotetext{
${ }^{1}$ We adopt the notion as in $[6]$ and $[10]$.

${ }^{2}$ There is a difference for the definition of the RR Page D0 charge between [8] and [1] by $C_{1}(T D) / 2$.

${ }^{3}$ Some related calculations can also be found in [12].
} 
boundary state in $S U(2)$ group manifold. We construct the dual for the $n$-th Cardy boundary state, which corresponds to the BPS D3-brane configuration with $z_{\max } \longrightarrow-\infty$ and opening angle $\left(\pi-\psi_{n}\right)$. As we show the dual Cardy boundary state $\mid k-n>_{C}$ can be obtained by rotating $\mid n>_{C}$ by $\pi$ in the plane $\left(y^{6}, y^{7}\right)$, from the correspondence this rotating operation is topologically equivalent to moving the upper D3-brane across to the other side of NS5-branes along $z$ direction. Since the BPS D3-brane configuration corrsponding to the $n$-th Cardy boundary state can be interpreted as that there are $n$ number of D1-strings which suspend between distant flat D3-brane and NS5-branes [9], by exploiting the correspondence between the Cardy boundary state and the D3-brane configuration in the full asymptotically flat geometry of the NS5-branes, we find that when the lower D3-brane passes through $k$ coincident NS5-branes, $k$ D-strings will be created, that is, the Hanany-Witten effect [13-22 in 10D curved background is encoded in the Cardy boundary states in $S U(2)$ group manifold. By rephrasing HananyWitten effect, we see that depending on which side the distant observer stays, he(she) will observe two sorts of the number of D-strings: either $n$ or $n-k$. As two different contraction to $e$ or $(-e)$ corresponds to the observer staying on the different side of NS5-branes from ten dimensional point of view, this explains why for the $n$-th spherical D2-brane (corresponding to the $n$ th Cardy boundary state) one observes two kinds of RR Page D0 charges. However, in the above physical processes, the RR Page D0 charge is not conserved due to Hanany-Witten effect [13. 22,

Now let us recall the background fields around a stack of $k$ coinciding flat NS5-branes which is given by 23]

$$
\begin{aligned}
d s^{2} & =d x^{2}+f d y^{2}, \\
e^{2 \Phi} & =g_{s}^{2} f \\
H_{k \ell m} & =-\epsilon_{k \ell m n} \partial_{n} f,
\end{aligned}
$$

where $\left\{x^{\mu}\right\}=\left(x^{0}, x^{1}, \cdots \cdots x^{5}\right)$ parameterize the directions along the NS5branes, $\left\{y^{m}\right\}=\left(y^{6}, y^{7}, y^{8}, y^{9}\right)$ are locations of the fivebranes. $\Phi$ and $H$ are the dilaton and NS 3-form field strength, and $g_{s}$ is the string coupling far 
from the branes. The harmonic function $f$ depends on the transverse space

$$
\begin{aligned}
& f=1+\frac{k \ell_{s}^{2}}{r^{2}} \\
& r=|\boldsymbol{y}|=\sqrt{k} \ell_{s} e^{\phi} .
\end{aligned}
$$

The background (2) and (3) interpolates between Minkowski space with constant $\Phi$ and a vanishing $H$, and a near-horizon region in which the geometry is an asymptotically linear dilaton solution

$$
\begin{aligned}
d s^{2} & =d x^{2}+k \ell_{s}^{2}\left(d \phi^{2}+d \Omega_{3}^{2}\right), \\
H & =2 k \ell_{s}^{2} \omega_{3},
\end{aligned}
$$

where $d \Omega_{3}^{2}$ and $\omega_{3}$ are the metric and volume form on the unit 3-sphere $S_{6789}^{3}$, and it describes the geometry of a throat

$$
R^{1,5} \times R_{\phi} \times S U(2) .
$$

The CFT describing the $S_{6789}^{3}$ is the $S U(2)$ WZW model at level k. The $S U(2)$ group element $g$ is related to the coordinates on $S_{6789}^{3}$ via

$$
g(\boldsymbol{y})=\frac{1}{|\boldsymbol{y}|}\left\{y^{6} \boldsymbol{1}+i\left(y^{8} \sigma_{1}+y^{9} \sigma_{2}+y^{7} \sigma_{3}\right)\right\} .
$$

The $S O(4) \sim S U(2)_{L} \times S U(2)_{R}$ global symmetry corresponding to rotations in the $R^{4}\left(y^{6}, y^{7}, y^{8}, y^{9}\right)$ acts on $g$ as $g \longrightarrow h_{L} g h_{R}$ where $h_{L(R)} \in S U(2)_{L(R)}$. Denoting the generators of $S U(2)_{L}\left(S U(2)_{R}\right)$ by $J^{a}\left(\bar{J}^{a}\right)$, one finds that $J^{3}-\bar{J}^{3}$ generates rotations in $\left(y^{6}, y^{7}\right)$ plane, while $J^{3}+\bar{J}^{3}$ is the generator of the rotations in $\left(y^{8}, y^{9}\right)$. For the following discussions, we choose the cylindrical coordinates $(z, \rho, \theta, \varphi)$

$$
\left(y^{6}, y^{7}, y^{8}, y^{9}\right)=(z, \rho \cos \theta, \rho \sin \theta \cos \varphi, \rho \sin \theta \sin \varphi)
$$

and spherical coordinates to replace $(z, \rho)$ by

$$
(z, \rho)=(r \cos \psi, r \sin \psi)
$$

where $\theta \in[0, \pi], \varphi \sim \varphi+2 \pi, \psi \in[0, \pi]$. 
To see what the physical significance of the Cardy boundary states [24] is in 10D supergravity background, we consider the BPS configuration in which the D3-brane is orthogonal to the NS5-branes, that is, preserving the $S O(3)_{789}$ symmetry. The typical feature for this BPS D3-brane configuration is that it includes an infinite tube which can be interpreted as D1-brane 99. The angle $\psi$ has a simple geometrical meaning: opening angle as shown in Fig.1

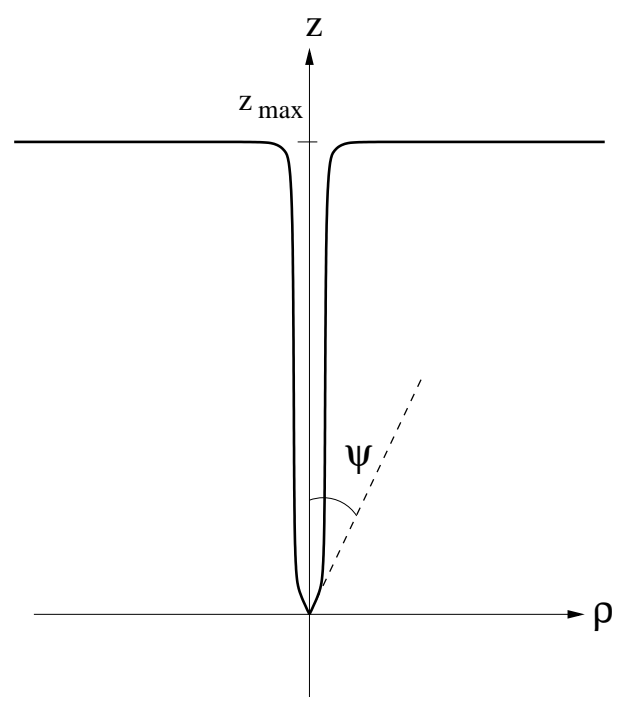

Figure 1: D3-brane profile for the fixed large $z_{\max }$ with opening angle $0<$ $\psi<\pi / 2$

Especially when $z_{\max } \longrightarrow \infty$, the infinite tube can be identified with D1branes [9].

In $S U(2)$ group manifold, the Cardy boundary states are given by 24

$$
\left|n>_{C}=\sum_{j=0}^{k} \frac{S_{n j}}{\sqrt{S_{0 j}}}\right| j>_{I}
$$

where $n=0,1, \cdots, k, \mid j>_{I}$ is the Ishibashi state [25] corrsponding to the chiral primary of spin $j / 2$, and

$$
S_{n j}=\sqrt{\frac{2}{k+2}} \sin \left(\frac{(n+1)(j+1) \pi}{k+2}\right)
$$


is the modular-transformation matrix. The Cardy boundary state $\mid n>_{C}$ describes the $n$-th spherical D2-branes on $S_{6789}^{3}$ labelled by [26]

$$
\psi_{n}=\frac{n \pi}{k}
$$

and possesses $n$ units of RR Page D0 charge which we shall interpret below as the number of D1-branes in 10D curved background.

In the background of $k$ coincident NS5-branes, the $n$-th Cardy boundary state corresponds to D3-brane intersecting with NS5-branes and taking the shape of a cone with opening angle $\psi_{n}$ in near-horizon region as illustrated in Fig. 2.

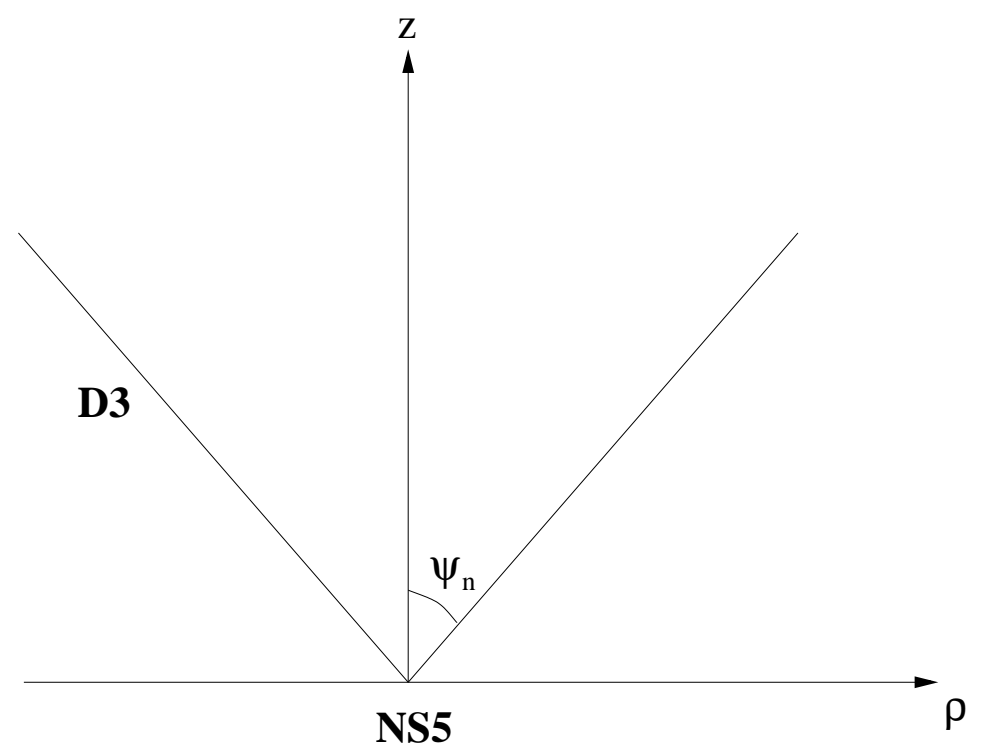

Figure 2: The D3-brane intersects with the NS5-branes in the near-horizon region with the form of a cusp

At the intersecting point $(z=\rho=0)$, there is a singularity, thus the Dirac-Born-Infeld effective action is not an adequate description of the test brane, however, in the present case of NS5-branes, we have an exact CFT in this region, with which we can do better, even work out Hanany-Witten effect which we shall show below.

Fig. 2 only describes D3-brane in the near-horizon region, if we recall the full asymptotically flat geometry of the multiple NS5-branes, the D3-brane 
configuration should take the form in Fig. 1, which can be interpreted as D1-strings suspending between the flat D3-brane and the NS5-branes [9].

What we have seen from the above discussion is that based on the numerical calcultion in 9] we observe that in 10D curved background, there is one to one correspondence between the $n$-th Cardy boundary state and the BPS D3-brane configuration with $z_{\max } \longrightarrow \infty$ and opening angle $\psi_{n}=n \pi / k$. The RR Page D0 charge of Cardy boundary state can be identified to the number of D1-branes which is depicted via the infinite throat [9].

We turn to construct the dual Cardy boundary state which corresponds to the D3-brane configuration with $z_{\max } \longrightarrow-\infty$ and opening angle $\left(\pi-\psi_{n}\right)$ as drawn in Fig. 3.

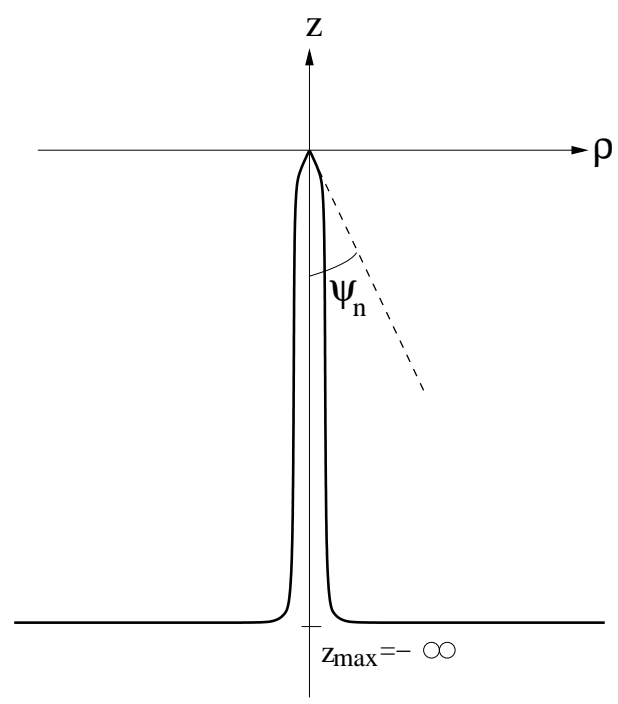

Figure 3: The shape of D3-brane corresponding to the dual Cardy boundary state

Since $J^{3}-\bar{J}^{3}$ and $J^{3}+\bar{J}^{3}$ are the generators of the rotations in $\left(y^{6}, y^{7}\right)$ and $\left(y^{8}, y^{9}\right)$ plane respectively, we construct the dual Cardy boundary state of $\mid n>_{C}$ by the rotation of $\pi$, i.e., $\left|n>_{C}^{D}=\exp \left\{i \pi\left(J_{0}^{3}-\bar{J}_{0}^{3}\right)\right\}\right| n>_{C}$. At first, we consider the bosonic case, and act the operator $\exp \left\{i \pi\left(J_{0}^{3}-\bar{J}_{0}^{3}\right)\right\}$ on Ishibashi state $\mid j>_{I}$ satisfying the boundary condition 25]

$$
\left(J_{n}^{3}+\bar{J}_{-n}^{3}\right)\left|j>_{I}=0, \quad\left(J_{n}^{ \pm}+\bar{J}_{-n}^{ \pm}\right)\right| j>_{I}=0
$$


which preserve maximal chiral Kac-Moody symmetry. One can show that the rotated boundary state $\exp \left\{i \pi\left(J_{0}^{3}-\bar{J}_{0}^{3}\right)\right\} \mid j>_{I}$ satisfies the same boundary condition (12) 1 . Since the general form of the Ishibashi state is

$$
\left|j>_{I}=\Pi_{i} J_{-n_{i}}^{a_{i}}\right| 0, j, j>\otimes\left(-\bar{J}_{-n_{i}}^{a_{i}}\right) \mid 0, j,-j>
$$

one can get

$$
\exp \left\{i \pi\left(J_{0}^{3}-\bar{J}_{0}^{3}\right)\right\}\left|j>_{I}=(-1)^{j}\right| j>_{I}
$$

and one has

$$
\begin{aligned}
\mid n>_{C}^{D} & =\left(\frac{2}{k+2}\right)^{1 / 4} \sum_{j=0}^{k} \frac{\sin [(n+1)(j+1) \pi /(k+2)]}{\sqrt{\sin [(j+1) \pi /(k+2)]}}(-1)^{j} \mid j>_{I} \\
& =\mid k-n>_{C}
\end{aligned}
$$

which indicates that the state corresponding to the D3-brane configuration in Fig. 3 can be obtained by rotation operator $\exp \left\{i \pi\left(J_{0}^{3}-\bar{J}_{0}^{3}\right)\right\}$ on the $n$-th boundary state which is consistent with the intuitive expectation.

Since the boundary condition (12) preserves the diagonal $S U(2)$ symmetry $g \longrightarrow h g h^{-1}$, the Cardy boundary state $\mid k-n>_{C}$ can also be constructed by acting the other rotation operator $\exp \left(2 \pi i J_{0}^{3}\right)\left(\operatorname{or} \exp \left(-2 \pi i \bar{J}_{0}^{3}\right)\right)$ on $\mid n>_{C}$, but with different bahavior in the supersymmetric generalization. The total $S U(2)$ current algebra of level $k+2$ is generated by the currents $J_{\text {total }}^{a}=J^{a}+j^{a}$, where $j^{a}=-\frac{i}{2} \epsilon^{a b c} \psi_{b} \psi_{c}$ is the contribution of the fermions. After a certain chiral rotation, we have

$$
\begin{gathered}
j_{0}^{3}=\sum_{n \geq 1}^{\infty}\left(\psi_{n+1 / 2}^{1} \psi_{-(n+1 / 2)}^{2}+\psi_{-(n+1 / 2)}^{1} \psi_{n+1 / 2}^{2}\right), \\
\bar{j}_{0}^{3}=-\sum_{n \geq 1}^{\infty}\left(\tilde{\psi}_{n+1 / 2}^{1} \tilde{\psi}_{-(n+1 / 2)}^{2}+\tilde{\psi}_{-(n+1 / 2)}^{1} \tilde{\psi}_{n+1 / 2}^{2}\right)
\end{gathered}
$$

for NS sector and

$$
\begin{aligned}
& j_{0}^{3}=\psi_{0}^{1} \psi_{0}^{2}+\sum_{n \geq 1}^{\infty}\left(\psi_{n}^{1} \psi_{-n}^{2}+\psi_{-n}^{1} \psi_{n}^{2}\right), \\
& \bar{j}_{0}^{3}=-\tilde{\psi}_{0}^{1} \tilde{\psi}_{0}^{2}-\sum_{n \geq 1}^{\infty}\left(\tilde{\psi}_{n}^{1} \tilde{\psi}_{-n}^{2}+\tilde{\psi}_{-n}^{1} \tilde{\psi}_{n}^{2}\right)
\end{aligned}
$$

\footnotetext{
${ }^{4}$ One can exploit the relation $e^{i \alpha J_{0}^{3}} J_{n}^{ \pm} e^{-i \alpha J_{0}^{3}}=e^{ \pm i \alpha} J_{n}^{ \pm}$.
} 
for $\mathrm{R}$ sector, and the minus sigh between $j_{0}^{3}$ and $\bar{j}_{0}^{3}$ is due to the opposite chiral rotation. The boundary condition for the fermionic part is

$$
\left(\psi_{n}^{a}-i \eta \tilde{\psi}_{-n}^{a}\right) \mid B_{\psi}, \eta>=0
$$

where $\eta= \pm 1, a=1,2,3$ and the fermionic boundary state takes the standard form as in flat space. Then the total boundary states of the $S U(2)$ WZW model factorize into

$$
\left|n, \pm>^{\text {susy }}=\right| n>_{C} \otimes \mid f, \pm>
$$

with

$$
|f, \pm>=| \mathrm{NS} \mathrm{NS}> \pm \mid \mathrm{R} \mathrm{R}>
$$

where $\mid f, \pm>$ denote the fermionic boundary states for the brane and antibrane.

In the supersymmetric case, there are two sorts of the rotation operators given by

$$
\mathcal{O}_{1}=\exp \left[i \pi\left(J_{0}^{3}-\bar{J}_{0}^{3}\right)+i \pi\left(j_{0}^{3}-\bar{j}_{0}^{3}\right)\right], \quad \mathcal{O}_{2}=\exp \left[2 \pi i\left(J_{0}^{3}+j_{0}^{3}\right)\right] .
$$

After the straightforward calculation, we have

$$
\mathcal{O}_{1}|n, \pm>=| k-n, \pm>, \quad \mathcal{O}_{2}|n, \pm>=| k-n, \mp>
$$

which indicates that $\mathcal{O}_{1}$ operator retains (anti)brane as (anti)brane, while $\mathcal{O}_{2}$ operator reverses (anti) brane into (brane) anti-brane due to the fermionic zero mode in the RR sector which changes the sign of the boundary state $\mid \mathrm{R} R>$.

In [8], it was argued that there should exist a finite translation operator which moves D0-branes from $e$ to $(-e)$ and maps the Cardy boundary state $\mid 0,+>^{\text {susy }}$ to $\mid k / 2,->^{\text {susy }}$. Here we have explicitly constructed the operator $\mathcal{O}_{2}$ which maps $\mid n, \pm>^{\text {susy }}$ to $\mid k-n, \mp>^{\text {susy }}$, thus our construction confirms their conjecture. 


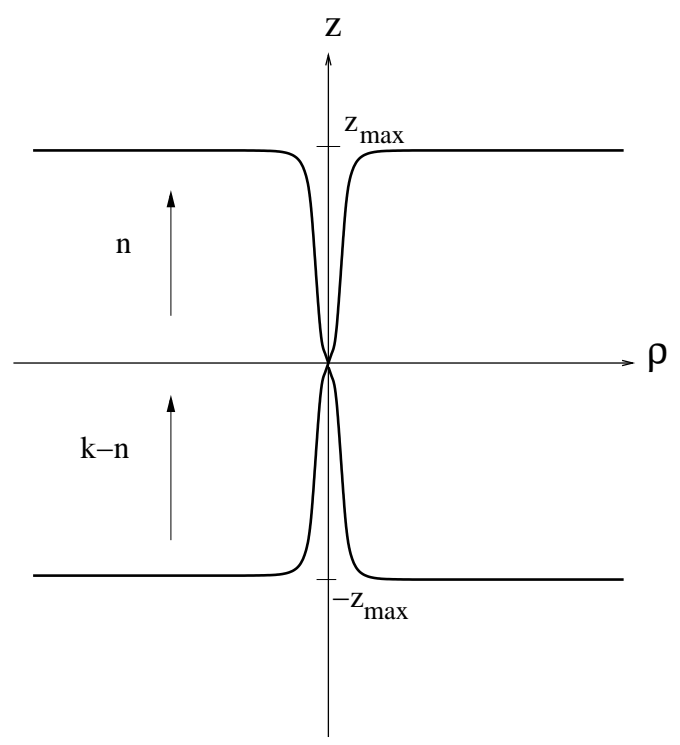

Figure 4: Two D3-branes with the same shape but placed on the opposite side of NS5-branes along $z$ direction, which corresponds to pair of Cardy boundary states $\mid n>_{C}$ and $\mid k-n>_{C}$

We consider the pair of the Cardy boundary states $\mid n>_{C}$ and $\mid k-n>_{C}$, whose RR Page D0 charges are $n$ and $k-n$. In Fig.4, the D3-brane configuration corresponding to the pair of Cardy boundary states $\mid n>_{C}$ and $\mid k-n>_{C}$ is portrayed円.

For definiteness, we assume that D-strings extend along $z$ direction, that is, for the lower D3-brane, $k-n$ D-strings emanate from the lower flat D3brane and terminate on NS5-branes, while for the upper D3-brane, $n$ Dstrings direct away from NS5-branes and end to the upper flat D3-brane. When we define the D-strings emanate from NS5-brane to their own D3branes, we see there are $n$ and $n-k$ D-strings for the upper and lower D3-branes 5 which is drwan in Fig. 5.

\footnotetext{
${ }^{5}$ In Fig.4, we do not mean we treat with the bound state by gluing together two BPS D3-brane solutions (corresponding to the Cardy boundary states $\mid n>_{C}$ and $\mid k-n>_{C}$ ). Here we mean that when $t \rightarrow-\infty$, the D3-brane locates at $z_{\max } \rightarrow-\infty$, and when $t \rightarrow \infty$, the lower D3-brane after moving across the NS5-branes finally stays at $z_{\max } \rightarrow \infty$.

${ }^{6}$ We have reversed the direction of the D-strings suspending between the lower flat D3-brane and the NS5-branes. Originally, they extend along $z$ direction and the number
} 


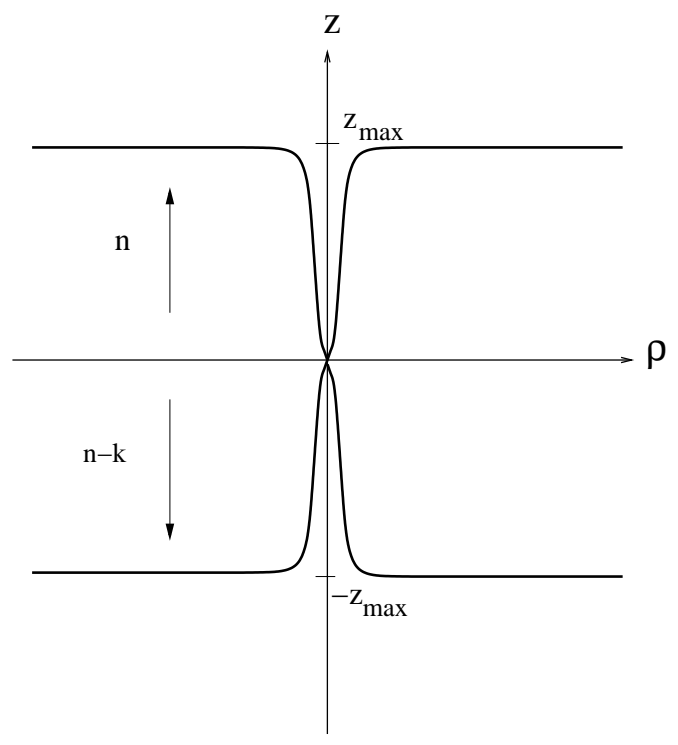

Figure 5: The D-strings emanate from NS5-branes to their own D3-branes, and the numbers of $n$ and $n-k$ indicate the number of the D-strings for each type.

As the Cardy state $\mid k-n>_{C}$ can be obtained by rotating $\mid n>_{C}$ by $\pi$ in the plane $\left(y^{6}, y^{7}\right)$, similarly the lower D3-brane can be obtained from the upper D3-brane by the same rotation [9], but this rotating operation is topologically equivalent to moving the upper D3-brane across to the other side of NS5-brane along $z$ direction. From the correspondence between Cardy boundary state and the D3-brane configuration in the full asymptotically flat geometry of the NS5-branes, we see in Fig. 5 that when the lower D3brane passes through the $k$ coinciding NS5-branes, $k$ D-strings are created. In other words, the Hanany-Witten effect can be read off from the Cardy boundary states through the above correspondence, especially the number of the created (or annihilated) D-strings is completely determined by the Cardy boundary state $\mid n>_{C}$ and its dual $\mid k-n>_{C}$ !

Since the intrinsic direction of the D1-strings is defined as they emanate from NS5-branes to their own D3-brane, the above string creation effect can be rephrased as that depending on which side the distant observer stays, he of D-strings is $k-n$, after reversion we denote the number of D-strings as $n-k$. 
(she) will measure two sorts of the number of D-strings: either $n$ or $n-k$. In $S U(2)$ WZW model, the spherical D-branes can be contracted either to the group unit $e$ along the 3 -ball $\Gamma$ or to the opposite pole $(-e)$ of $S_{6789}^{3}$ along the 3-ball $\Gamma^{\prime}$ [8], [11]. Two ways of computing RR Page D0 charge of a spherical D2-brane result in two RR Page charge $n$ or $n-k$, and the flux is only determined modulo $k$ if we demand in physical process the RR Page D0 charge is reserved. In [8], this peculiar feature of the RR Page D0 charge was expected to be related to the fact that $H$-field belongs to a nontrivial cohomology class. However, in the present context, we find that two sorts of RR Page charge is due to two different relative position between the observer and the measured D3-brane. To be more precise, suppose that D3-brane is placed on the upper side of the NS5-branes, the distant observer can stay on upper side and the measured RR Page charge is $n$, but he (she) can also choose to stay on lower side and the observed RR Page D0 charge is $n-k$, which can be interpreted as $D$-string creation in the full NS5-brane geometry】. Actually, in the above physical process, RR Page D0 charge is not a conserved quantity. When we define RR Page D0 charge modulo $k$, we effectively erase D-string creation effect in 10D curved space. If we use brane source charge instead of Page charge, the Hanany-Witten effect is also removed.

In summary, we have observed the correspondence between the Cardy boundary states in $S U(2)$ group manifold and the BPS D3-brane configuration preserving the $S O(3)_{789}$ symmetry in the full asymptotically flat geometry of the $k$ coincident NS5-branes. The dual Cardy boundary state has been constructed by acting the rotation operator on $\mid n>_{C}$, which rotates $\pi$ in $\left(y^{6}, y^{7}\right)$ plane. Exploiting the correspondence and the dual Cardy boundary state, we have found that the Hanany-Witten effect, the generalization of the string creation mechanism for the case of D0/D8-branes [14]-[21], can be

\footnotetext{
${ }^{7}$ In D0/D8 case, the number of the fundamental strings suspending between D0- and D8-brane is proportional to $|z| / 2 z$ and dependent on how to choose positive direction for z. Usually one defines the positive direction for $\mathrm{z}$ as that it extends from D8-brane to the observer, and the observer will measure $+1 / 2 \mathrm{~F}$-string if D0-brane is at the observer's side, but $-1 / 2 \mathrm{~F}$-string if at the opposite side [18].
} 
induced from the Cardy boundary states. By rephrasing Hanany-Witten effect, we have shown that due to the contraction to $e$ or $(-e)$, the two different RR Page D0 charges for the $n$-th spherical D2-brane in $S U(2)$ WZW model can be interpreted as string creation in the background of the $k$ coinciding NS5-branes.

\section{Acknowledgements}

We would like to thank P.M. Ho and M. Kreuzer for valuable discussions. The work of T.K. is supported in part by Scientific Grants from the Ministry of Education (grant Nr., 09640353). The work of J.-G. Z. is supported in part by the Austrian Research Funds FWF under grant Nr. M535-TPH.

\section{References}

[1] C. Bachas, M. Douglas and C. Schweigert, "Flux stabilization of Dbranes" hep-th/0003037.

[2] J. Pawelczyk, "SU(2) WZW D-branes and their noncommutative geometry from DBI action" hep-th/0003057.

[3] W. Taylor, "D2-branes in B fields" hep-th/0004141.

[4] A. Kling, M. Kreuzer and J.-G. Zhou, "SU(2) WZW D-branes and quantized worldvolume $U(1)$ flux on $S^{2} "$, hep-th/0005148.

[5] A. Alekseev, A. Mironov and A. Morozov, "On B-independence of $R R$ charges", hep-th/0005244.

[6] D. Marolf, "Chern-Simons terms and three notions of charge", hepth/0006117.

[7] C. Stanciu, "A note on D-branes in group manifolds: flux quantization and D0-charge", hep-th/0006145.

[8] A. Alekseev and V. Schomerus, " $R R$ charges of D2-branes in the $W Z W$ model", hep-th/0007096. 
[9] O. Pelc, "On the quantization constraints for a D3 brane in the geometry of NS5 branes", hep-th/0007100.

[10] D. Marolf, "Half-branes, singular brane intersections and Kaluza-Klein reduction", hep-th/0007171.

[11] J.M. Figueroa-Q'Farrill and S. Stanciu, "D-brane charge, flux quantization and relative (co)homology", hep-th/0008038.

[12] P.M. Llatas, A.V. Ramallo and J.M. Sanchez de Santos, "World-volume solitons of the D3-brane in the background of $(p, q)$ five-branes ", hepth/9912177.

[13] A. Hanany and E. Witten, "Type IIB superstrings, BPS monopoles, and three dimensional gauge dynamics ", Nucl. Phys. B 492 (1997) 152, hep-th/9611230.

[14] U. Danielsson, G. Ferretti and I.R. Klebanov, "Creation of fundamental strings by crossing D-branes", Phys. Rev. Lett. 79 (1997) 1984, hepth/9705084.

[15] O. Bergman, M.R. Gaberdiel and G. Lifschytz, "Branes, orientifolds and the creation of elementary strings", Nucl. Phys. B 509 (1998) 194, hep-th/9705130.

[16] S.P. de Alwis, "A note on brane creation", Phys. Lett. B 388 (1997) 720 , hep-th/9706142.

[17] P. Ho and Y. Wu, "Brane creation in M(atrix) theory", Phys. Lett. B 420 (1998) 43, hep-th/9708137.

[18] N. Ohta, T. Shimizu and J.-G. Zhou, "Creation of fundamental string in M(atrix) theory", Phys. Rev. D 57 (1998) 2040, hep-th/9710218.

[19] T. Nakatsu, K. Ohta, T. Yokono and Y. Yoshida, "A proof of brane creation via M-theory", Mod. Phys. Lett. A 13 (1998) 293, hepth/9711117. 
[20] Y. Yoshida, "Geometrical analysis of brane creation in M-theory", Prog. Theor. Phys. 99 (1998) 305, hep-th/9711177.

[21] T. Kitao, N. Ohta and J.-G. Zhou, "Fermionic zero mode and string creation between D4-branes at angles", Phys. Lett. B 428 (1998) 68, hep-th/9801135.

[22] C.G. Callan, A. Guijosa and K.G. Savvidy, "Baryons and string creation from the fivebrane worldvolume action", Nucl. Phys. B 547 (1997) 127, hep-th/9810092.

[23] C.G. Callan Jr., J.A. Harvey and A. Strominger, "Supersymmetric string solitons", Lectures given by C. Callan and J. Harvey at the 1991 Trieste spring school "String theory and quantum gravity", hep-th/9112030.

[24] J.L. Cardy, "Boundary conditions, fusion rules and the Verlinde fromula ", Nucl. Phys. B 324 (1989) 581.

[25] N. Ishibashi, "The boundary and crosscap states in conformal field theories", Mod. Phys. Lett. A4 (1989) 251.

[26] A.Yu. Alekseev and V. Schomerus, "D-branes in the WZW model", Phys. Rev. D 60 (1999) 061901, hep-th/9812193. 